GRADIATION\&APPLICATIONS

ISSN 2466-4294 (online) | rad-journal.org

Vol. 3 | Issue 2 | pp. 117-122, 2018

doi: 10.21175/RadJ.2018.02.019

Original research paper

\title{
ANNEALING STUDIES ON IRRADIATED P-TYPE SILICON STRIP SENSORS DESIGNED FOR THE ATLAS PHASE II TRACKING DETECTOR*
}

\author{
L. Diehl ${ }^{* *}$, L.Wiik-Fuchs, R. Mori, M. Hauser, K. Jakobs, U. Parzefall
}

Albert-Ludwigs-Universität Freiburg, Freiburg im Breisgau, Germany

\begin{abstract}
In 2025, the Large Hadron Collider (LHC) will be upgraded to the High Luminosity (HL-)-LHC. This will challenge the silicon strip detector performance with very high fluences and long operation time. Sensors have been designed to survive severe radiation damage as demonstrated by electrical tests and charge collection measurements. Besides that, it is important to predict and understand the long-term evolution of the sensor properties. In this paper, detailed studies on the annealing behavior of ATLAS 12 strip detectors designed by the ITK Strip Sensor Working Group and irradiated with fluences between $5 \cdot 10^{13}$ and $2 \cdot 10^{15} n_{e q} / \mathrm{cm}^{2}$ are presented. During the annealing time at $23^{\circ} \mathrm{C}$ and $58.5^{\circ} \mathrm{C}$ systematic charge collection, leakage current and impedance measurements have been carried out until breakdown or the appearance of charge multiplication. The phenomenon of charge multiplication in high irradiated sensors after long annealing times has been investigated with respect to dependencies on temperature and bias voltage cycling. The difference in the annealing behavior between the two temperatures has been analyzed and compared to similar measurements on n-type sensors and with a theoretical model. For sensors with fluences below $3 \cdot 10^{14} n_{e q} / \mathrm{cm}^{2}$ the effective doping concentration could be extracted from the impedance measurements and was compared with a theoretical model. The results show that ATLAS12 sensors anneal similarly to the previously designed ATLASO7 and the behavior is well described by the theoretical model. Nevertheless, a significant difference in the time constant of the beneficial and reverse annealing with respect to previous n-type sensors has been reported.
\end{abstract}

Key words: Silicon strip sensor, ATLAS Upgrade, charge collection, annealing, charge multiplication

\section{INTRODUCTION}

The HL-LHC [1] will start data-taking in 2026. The silicon sensors in the tracking detector and their operation will face enormous challenges due to the unprecedented radiation fluences that are created by a total integrated luminosity of up to $4000 \mathrm{fb}^{-1}$. Therefore, the current Inner Tracker will be exchanged with an all-silicon Inner Tracker [2] by the ATLAS collaboration [3]. The HL-LHC will have shutdown periods on a yearly basis for machine maintenance, in which the silicon sensors cannot be cooled the entire time. The electrical properties of silicon sensors are significantly changed by hadron radiation. The changes are caused by silicon bulk defects, which are subject to a complex migration progress, called annealing. Annealing has a strong temperature and time dependence. Hence it can be accelerated or decelerated by using a higher or lower annealing temperature, as can be concluded from the Hamburg model [4]. For time reasons, a scaling factor from the Hamburg model is usually assumed and annealing studies are conducted at elevated temperatures in general, mostly around $60^{\circ} \mathrm{C}$, and measurements are then corrected to the equivalent annealing time of several years at room temperature. Due to their superior radiation hardness [5,6], sensors with $\mathrm{n}^{+-}$ readout, most commonly realized with a p-bulk, are mandatory for the HL-LHC, while most previous annealing studies were carried out with n-type sensors. Previous studies on the annealing behavior of p-type silicon can for instance be found in $[7,8,9]$. This study presents the annealing behavior of ATLAS12 [10] sensors designed by the ATLAS collaboration and produced by Hamamatsu Photonics [11]. It includes sensors irradiated to different fluences between $5 \cdot 10^{13}$ and $2 \cdot 10^{15} \mathrm{neq} / \mathrm{cm}^{2}$. To assess the temperature dependence during the long-term annealing, the sensors in this study were separated in two groups and either annealed at room temperature or $58.5^{\circ} \mathrm{C}$. The sensor behavior was evaluated after each annealing step by measuring charge collection and leakage current and in some cases the impedance. Some sensors showed charge multiplication. Furthermore, a detailed investigation on the difference in the annealing behavior of sensors annealed at room temperature and $58.5^{\circ} \mathrm{C}$ is presented and a comparison with the Hamburg model is given.

\section{DEVICES UNDER Test}

The sensors evaluated in this study are miniature $(1 \mathrm{~cm} \times 1 \mathrm{~cm})$ sensors from the ATLAS12 prototype production, manufactured by Hamamatsu Photonics [11]. These are single-sided p-bulk standard float-zone sensors with n-type implants, cut from six-inch wafers with a nominal thickness of $310 \pm 20 \mu \mathrm{m}$. The sensors have a bulk resistivity of about $3.5 \mathrm{k} \Omega \mathrm{cm}$, hence the

\footnotetext{
* This paper was presented at the Sixth International Conference on Radiation and Applications in Various Fields of Research (RAD 2018), Ohrid, Macedonia, 2018.

**eena diehl@gmx.de
} 
depletion voltage is on the order of $350 \mathrm{~V}$. Each miniature sensor has a strip pitch of $74.5 \mu \mathrm{m}$ such that each sensor contains 103 strips with a length of $0.8 \mathrm{~cm}$. The n-type strips are connected to the bias rail via poly-silicon resistors implanted in the sensor.

Different interstrip isolation schemes are implemented, using either uniform p-spray, p-stop implants or a combination of both. This is to guarantee strip isolation during the lifetime of the sensors and further to limit radiation-induced surface damage while maintaining a low inter-strip capacitance. Previous studies evaluating the performance of these sensors under irradiation are found in $[12,13,14]$.

To determine the behavior of the sensors after radiation exposure in doses similar to the expected fluences at the HL-LHC the strip sensors were irradiated with protons at the Irradiation Center in Karlsruhe using a $23 \mathrm{MeV}$ proton beam.

The sensors used for this study were irradiated to fluences of $5 \cdot 10^{13}, 1 \cdot 10^{14}, 2 \cdot 10^{14}, 3 \cdot 10^{14}, 5 \cdot 10^{14}, 1 \cdot 10^{15}$ and $2 \cdot 10^{15} \mathrm{n}_{\mathrm{eq}} / \mathrm{cm}^{2}$.

\section{EXPERIMENTAL SETUP ANd Signal ANALYSis}

\subsection{Setups}

The charge collection measurements were performed using a ${ }^{\circ} \mathrm{Sr}$ source emitting electrons close to Minimal Ionizing Particles (MIPs). Two scintillators positioned behind the device under test are used to trigger particles traversing the sensor. The use of the coincidence of two scintillators as a trigger further helps to reject events arising from low energetic particles and photons created by bremsstrahlung. The measurements are done in a humidity and temperature-controlled environment, using a freezer flushed with nitrogen. They were conducted with a relative humidity below $10 \%$ and at temperatures between $-14^{\circ} \mathrm{C}$ and $-30^{\circ} \mathrm{C}$, necessary to suppress the leakage current, which was recorded during the measurements and scaled to a uniform temperature of $\mathrm{O}^{\circ} \mathrm{C}$ afterwards. The data acquisition was done using the ALIBAVA system [15], which is based on LHC compatible $40 \mathrm{MHz}$ analogue electronics built around the Beetle chip [16]. The impedance measurements were also conducted in a humidity-controlled freezer at temperatures between $-12^{\circ} \mathrm{C}$ and $-20^{\circ} \mathrm{C}$. The sensors were connected to an LCR-meter and measured at several frequencies.

In both measurements the sensors were biased, and the leakage current was measured using a Keithley 237. The sensors annealed at $58.5^{\circ} \mathrm{C}$ were kept in a dedicated temperature control oven with a temperature stability of $58.5 \pm 1^{\circ} \mathrm{C}$. The other set of sensors was annealed at a room temperature of $23 \pm 1^{\circ} \mathrm{C}$ in a temperature controlled clean room.

\subsection{Signal Analysis}

The signal in the charge collection measurements was analyzed using a customized software package including an iterative common mode and pedestal subtraction algorithm. Channels showing excessive noise behavior were additionally masked. Events were rejected if their path did not originate from the path between the source and the scintillators. Furthermore events within a time window of $10 \mathrm{~ns}$ around the signal peak are selected to allow high statistics, leading to a small signal underestimation of less than $5 \%$. The charge is determined using a clustering algorithm with a seed channel signal exceeding 3.5 the noise and a neighbours having a signal at least 1.8 times larger than noise, to exclude spurious hits and noise contributions to the signal. The most probable value of a Landau function convoluted with a Gaussian to the the number of events plotted against the measured charge is used for further analysis.

Impedance measurements, or parallel capacitance and resistance versus voltage and frequency, have been monitored in order to observe the changes. For high fluences the measurements showed a strong influence of the defects reaction times and its changes with annealing, but a thorough analysis of these data goes beyond the aim of this manuscript. Therefore, the analysis on the capacitance was limited and a single frequency reasonably for representing its steady state value was chosen after evaluating the frequency behavior. In this way the effective doping concentration can be extracted as following. For a dedicated frequency of $1 \mathrm{kHz}$ the inverse of the squared capacitance was plotted against the bias voltage. With a linear fit the slope could be determined and the effective doping concentration calculated using the formula

$$
\frac{1}{C^{2}}=\frac{1}{A^{2}} \frac{2 V}{\varepsilon q N_{e f f}},
$$

with the Capacitance $\mathrm{C}$, the active sensor area $\mathrm{A}$, the elementary charge $\mathrm{q}$ and the electric field constant $\varepsilon$ [17]. All measurements were taken at various bias voltages ranging from $100 \mathrm{~V}$ to $1100 \mathrm{~V}$.

\section{LONG TERM ANNEALING BEHAVIOR}

The initial charge collection measurement of the sensors was conducted without any additional annealing other than caused by transportation and handling of the sensors after irradiation. The duration of the annealing steps was increased from initially 30 min to $10000 \mathrm{~min}$ at $58.5^{\circ} \mathrm{C}$, to account for the logarithmic timescale of the behavior. All sensors were annealed either until break-down or the occurring of charge multiplication. The maximum annealing time is found for the sensor irradiated to $2 \cdot 10^{14} \mathrm{n}$ eq $/ \mathrm{cm}^{2}$ with $40000 \mathrm{~min}$ of accelerated annealing, corresponding to more than seven years at room temperature. Collected charge (top) and leakage current (bottom) of this sensor are shown in Figure 1 for different voltages in dependence of the annealing time. The collected charge of the sensors follows the expected behavior from the Hamburg model [17]. In the beneficial annealing phase of up to $300 \mathrm{~min}$, the collected charge slightly increases. Afterwards, in the reverse annealing phase, the charge deteriorates for voltages below 800 $\mathrm{V}$, showing that the space charge region depth for the same voltage decreases with time, indicating the increase of the effective doping concentration and thus 
the need of higher voltages to reach full depletion. For bias voltages higher than $1000 \mathrm{~V}$ no decline is seen, in contrary for annealing times longer than $1000 \mathrm{~min}$ a slight increase is visible. This might be attributed to the onset of charge multiplication, as suggested in previous studies [18], which is however not fully reached due to the low fluence. The leakage current decreases throughout the whole annealing time, as long as no onset of charge multiplication or breakdown occurs. In the plots the last points at the highest voltages are already missing due to the start of breakdown.
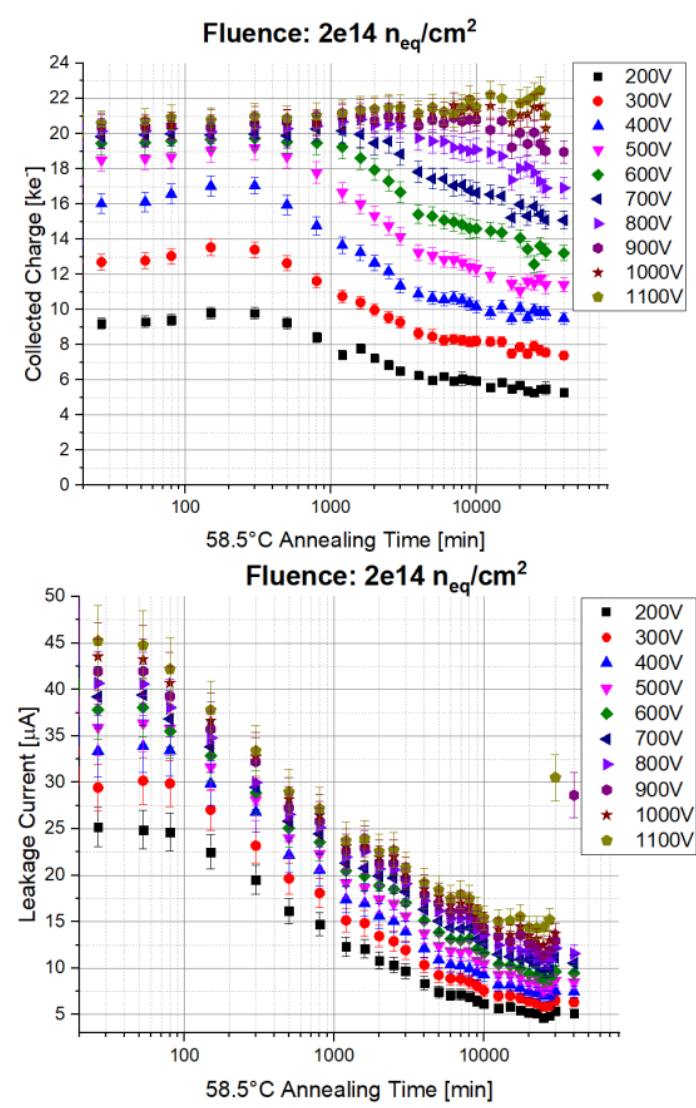

Figure 1. Most probable value of the collected charge (top) and leakage current (bottom) of the sensor irradiated to $2 \cdot 10^{14}$ $\mathrm{n}_{\mathrm{eq}} / \mathrm{cm}^{2}$ as a function of the annealing time for different bias voltages.

In Figure 2, the effective doping concentration of this sensor extracted from the capacitance measurements at a frequency of $1 \mathrm{kHz}$ is shown in dependence of the annealing time. At early time the effective doping concentration decreases - beneficial annealing, while at later times it significantly increases - reverse annealing. After long annealing times the measurements start to lose accuracy, but they hint at a saturation of the doping concentration. The full depletion voltage is proportional to the effective doping concentration, which in turns means that at given voltage the collected charge is smaller for higher effective doping concentrations. Using the equation

$$
\Delta N_{e f f}=N_{0} e^{-\frac{t}{\tau}}+N_{C}+N_{\infty}\left(1-e^{-k t}\right)_{(2)}
$$

from the Hamburg model [17], the data can be fitted to extract information about the time constants.

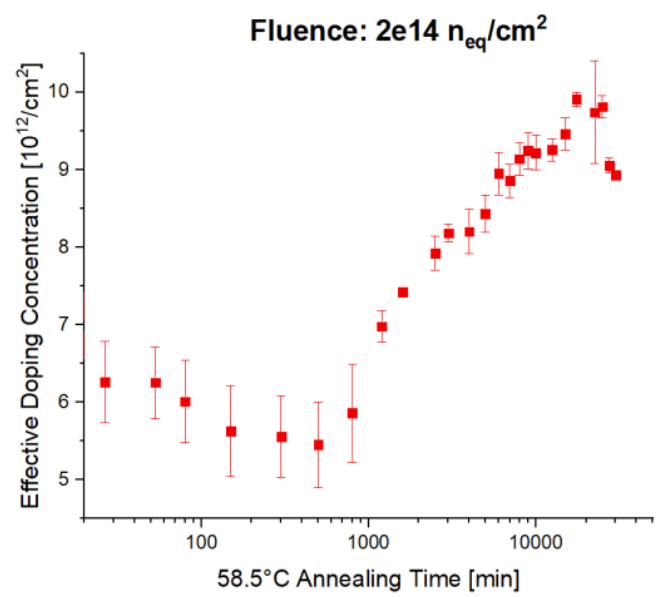

Figure 2. Effective doping concentration of the sensor irradiated to $2 \cdot 10^{14} \mathrm{n}_{\mathrm{eq}} / \mathrm{cm}^{2}$ as a function of the annealing time.

The fitted data for the sensors irradiated to $1 \cdot 10^{14}$ and $3 \cdot 10^{14} \mathrm{n}_{\text {eq }} / \mathrm{cm}^{2}$ annealed either at room temperature or $58.5^{\circ} \mathrm{C}$ are shown in Figure 3. They all exhibit the expected behavior and the extracted values from the fits are in the expected range. The sensors annealed at room temperature reaches the minimum of the effective doping concentration, which marks the end of the beneficial annealing, approximately 100 times slower than at $58.5^{\circ} \mathrm{C}$. This is the first hint on the scaling factor between the two temperatures. We also notice that ATLASo7 sensors behave in a similar way and anneal with the same time constants as the ATLAS12.

The previously known scaling factor between these two temperatures could be calculated using the formula

$$
t_{T_{\text {ref }}}=t_{T_{\text {ann }}} \cdot \exp \left(\frac{E_{A}}{k_{B}} \cdot\left(\frac{1}{T_{\text {ref }}}-\frac{1}{T_{\text {ann }}}\right)\right)
$$

from studies carried out with n-type silicon sensors [17]. The value used there for the activation energy $E_{A}$ is $1.31 \pm 0.03 \mathrm{eV}$ for the reverse annealing part. Considering the possible temperature fluctuations of $1^{\circ} \mathrm{C}$ the old scaling factor is $245 \pm 70$.

To get precise information on the temperature dependence, the time constants were extracted from the fits of damage parameter and effective doping concentration and compared with the literature values.

At a fluence of $3 \cdot 10^{14} \mathrm{n}_{\mathrm{eq}} / \mathrm{cm}^{2}$ the determination of the effective doping concentration from the capacitance measurements starts to lose accuracy because the slope is not anymore linear, but it is still reasonable. At higher fluences the presence of defects with emission times comparable with the AC signal periods, which are not participating in the charge movement, is not anymore negligible. It is impossible to extract the steady state value of the capacitance and the effective doping concentration. For the radiation dose of $2 \cdot 10^{14} \mathrm{n}_{\text {eq }} / \mathrm{cm}^{2}$ only one sample was available, 
which was annealed at $58.5^{\circ} \mathrm{C}$ to study extremely long annealing times.

Besides the effective doping concentration, another parameter which depends on the time constants of the annealing process is the leakage current. Leakage current is proportional to the fluence with the proportionality factor $\alpha$ - leakage current damage constant - calculated as [17]

$$
\alpha(t)=\frac{\Delta I}{\Phi_{e q} V}
$$

where $\Delta \mathrm{I}$ is the increment off the leakage current from the unirradiated value and $\Phi$ is the irradiation fluence; it can be described by [17]

$$
\alpha=\alpha_{I} e^{-\frac{t}{\tau}}+\alpha_{0}-\beta \ln (t)
$$

where $\tau$ is the annealing time constant. Figure 4 shows the damage parameter $\alpha$ along with the fits for the sensors irradiated with $1 \cdot 10^{14}$ (upper) and $3 \cdot 10^{14}$ (lower) $\mathrm{n}_{\mathrm{eq}} / \mathrm{cm}^{2}$ in dependence on the annealing time. For the $1 \cdot 10^{14} \mathrm{n}_{\mathrm{eq}} / \mathrm{cm}^{2}$ irradiated samples the leakage currents are significantly different, but the annealing times are similar. It is possible that some of these sensors had a scratch on the surface or suffered from mechanical stress, which would result in a larger leakage current but without an impact on the annealing time constant.

With the extracted time constants and assuming the same Boltzmann behavior as described in [17] we can calculate the activation energy and the frequency factor of the annealing process, using:

$$
\frac{1}{\tau}=k e^{\frac{E_{a}}{k_{B} T}}
$$

The corresponding Arrhenius relations for effective doping concentration and leakage current are shown in Figure 5

The measured values for the ATLAS12 and ATLASo7 sensors deviate from the expected values. It is clear that if the beneficial annealing of the effective doping concentration is slightly slower, as also evident by comparing figure 3 with the similar plot in [17], the reverse annealing is much faster at room temperature. The beneficial annealing time constants from the leakage current (damage parameter) are scattered due to the sensible fit of the leakage current. From fits of the effective doping concentration the time constants give an activation energy of $1.13 \pm 0.98 \mathrm{eV}$ and $1.09 \pm$ $0.18 \mathrm{eV}$, and a frequency factor of $2.80 \cdot 10^{13} \pm 1.08 \cdot 10^{15}$ $\mathrm{S}^{-1}$ and $4.22 \cdot 10^{11} \pm 3.09 \cdot 10^{12} \mathrm{~s}^{-1}$ for the beneficial and reverse annealing, respectively. The beneficial annealing gives values in agreement with the phonon frequency of a single jump of $1 \cdot 10^{13} \mathrm{~s}^{-1}$ and the activation energy reported in [17], while lower values for the reverse annealing are found. A thorough investigation of these discrepancies can be done utilizing defect spectroscopy techniques.
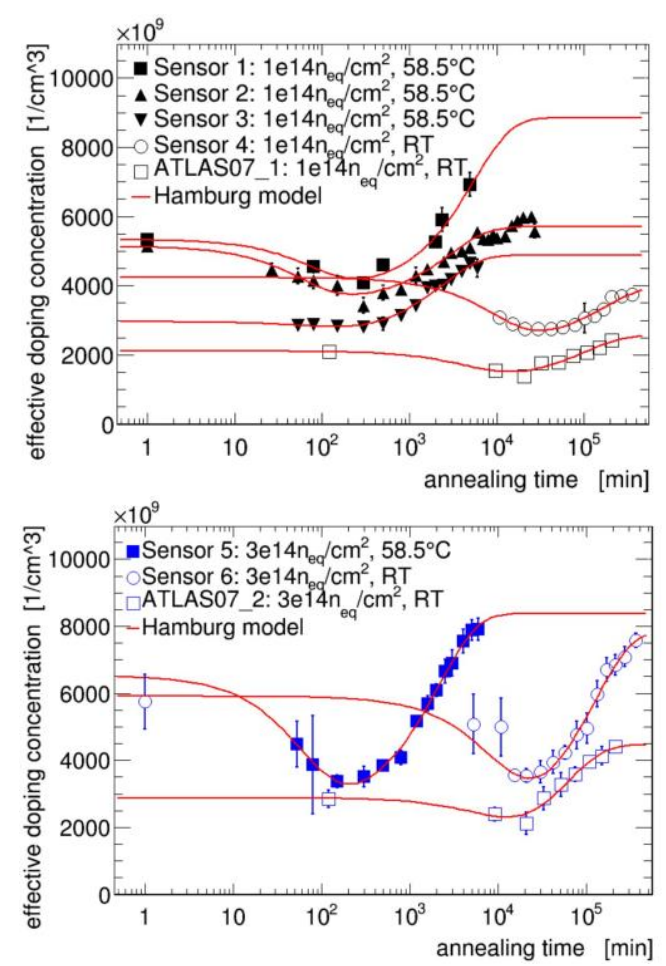

Figure 3. Effective Doping Concentration of the sensors irradiated with $1 \cdot 10^{14}$ and $3 \cdot 10^{14} \mathrm{n}_{\mathrm{eq}} / \mathrm{cm}^{2}$ annealed at $58.5^{\circ} \mathrm{C}$ or room temperature depending on the annealing time fitted with the formula (2).
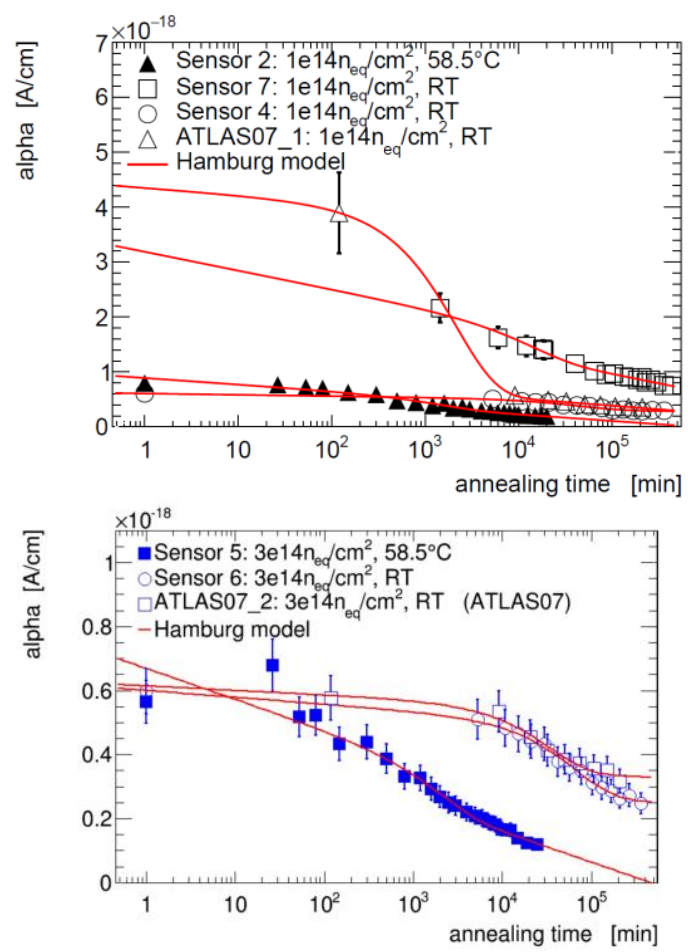

Figure 4. Damage parameter $\alpha$ of the sensors irradiated with $1 \cdot 10^{14}$ and $3 \cdot 10^{14} \mathrm{n}_{\mathrm{eq}} / \mathrm{cm}^{2}$ annealed at $58.5^{\circ} \mathrm{C}$ or room temperature depending on the annealing time fitted with the formula (4) 
L. Diehl et al., Annealing studies on silicon strip sensors, Rad. Applic., 2018, 3, 2, 117-122

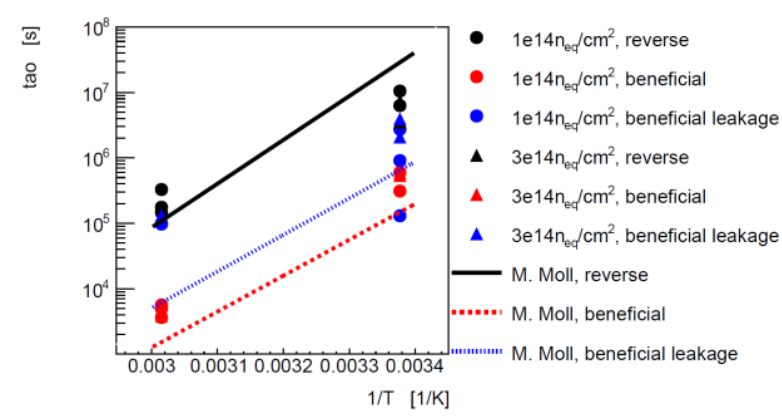

Figure 5. Time constant $\tau$ for fluences between $1 \cdot 10^{14}$ and $3 \cdot 10^{14} \mathrm{n}_{\text {eq }} / \mathrm{cm}^{2}$ depending on the inverse of the temperature compared with the literature values from [17]. Black and red

points correspond to the effective doping concentration.

The effective doping concentration determines the depleted volume for a given voltage and in turns it is directly correlated to the charge collection efficiency. Because of this, besides effective doping concentration and damage parameter fits a graphical determination of the scaling factor between the two temperatures with the charge collection was done.

The collected charge was plotted versus the annealing time and the time axis of the collected charge of the sensor annealed at room temperature was expanded until it corresponded to the one of the sensors annealed at $58.5^{\circ} \mathrm{C}$. In Figure 6, the collected charge depending on the annealing time at $58.5^{\circ} \mathrm{C}$ is shown.

This graphical determination was done using the reverse annealing part of five sensor sets with fluences between $5 \cdot 10^{13} \mathrm{n}_{\text {eq }} / \mathrm{cm}^{2}$ and $2 \cdot 10^{15} \mathrm{n}_{\text {eq }} / \mathrm{cm}^{2}$. The errors are estimated by the range in which the graphical scaling was approximately correct. The factors received are summarized in Table 1 . They all are between 100 and 110 , meaning that 1 minute at $58.5^{\circ} \mathrm{C}$ has the same annealing effects as 100-110 minutes at room temperature.

Considering the literature value of 245 , this means that the new ATLAS12 sensors anneal significantly faster at room temperature compared to $58.5^{\circ} \mathrm{C}$ than expected.

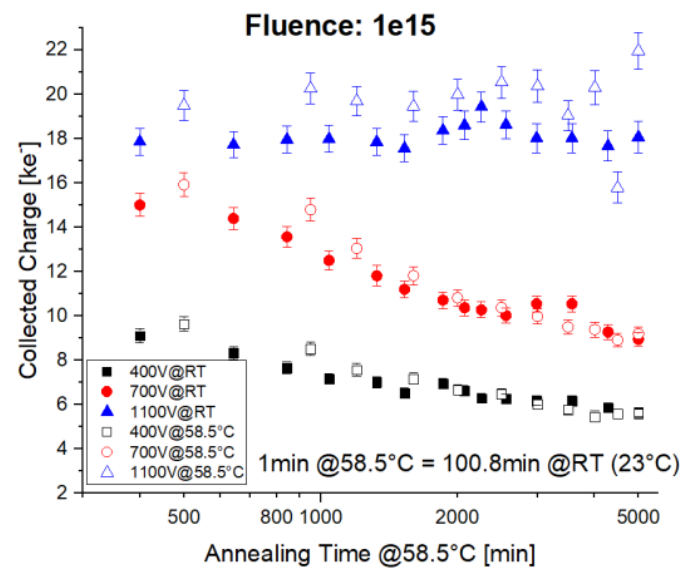

Figure 6. Collected charge of the sensors irradiated up to $1 \cdot 10^{15}$ $\mathrm{n}_{\mathrm{eq}} / \mathrm{cm}^{2}$ depending on the annealing time for three different voltages. The time axis of the room temperature annealed sensor was stretched until the course of the charge corresponded to the one of the $58.5^{\circ} \mathrm{C}$ annealed sample.
Table 1. Scaling factor extracted from leakage current or collected charge for different fluences

\begin{tabular}{|l|l|l|l|l|l|}
\hline $\begin{array}{l}\text { Fluence } \\
{\left[\mathrm{n}_{\text {eq }} / \mathrm{cm}^{2}\right]}\end{array}$ & $5 \cdot 10^{13}$ & $1 \cdot 10^{14}$ & $5 \cdot 10^{14}$ & $1 \cdot 10^{15}$ & $2 \cdot 10^{15}$ \\
\hline $\begin{array}{l}\text { Scaling factor } \\
\mathrm{k}\end{array}$ & $108 \pm 8$ & $101 \pm 15$ & $108 \pm 12$ & $101 \pm 9$ & $108 \pm 8$ \\
\hline
\end{tabular}

\section{CONCLUSION}

At the HL-LHC the operation conditions will challenge the silicon strip detectors both with high radiation doses, long operation times and temperature cycles between $-20^{\circ} \mathrm{C}$ and room temperature during maintenance periods. A thorough understanding of the room-temperature annealing is crucial for detector operations at the HL-LHC. A detailed study on the annealing behavior of ATLAS12 silicon strip sensors, irradiated with $23 \mathrm{MeV}$ protons to fluences between $5 \cdot 10^{13}$ and $2 \cdot 10^{15} \mathrm{n}_{\mathrm{eq}} / \mathrm{cm}^{2}$, has been performed. Changes of the detector behavior due to annealing have been evaluated by measuring the collected charge, the leakage current and the effective doping concentration throughout continuous annealing steps. The annealing was conducted either at room temperature $\left(23^{\circ} \mathrm{C}\right)$ or $58.5^{\circ}$ C. The measurements were performed in a temperature- and humidity-controlled environment until charge multiplication or a breakdown occurred.

To be able to investigate annealing processes in a short time, annealing studies are often conducted at higher temperatures, which is why a scaling factor is needed to predict the time it takes for these processes at room temperature.

The results of this study indicate that there are differences in the annealing of ATLAS12 sensors with respect to previously used n-type sensors. Although the general annealing behavior corresponds to the literature expectations, the temperature dependence of the effective doping concentration annealing appears to be different. Besides a difference in the annealing frequency also the activation of the physical process, that yields a scaling factor of 265 between $23^{\circ} \mathrm{C}$ and $58.5^{\circ} \mathrm{C}$, seems to different for the new p-type ATLAS12 sensors. The scaling factor derived in this study is approximately 100-110, with the new sensors showing a significantly faster reverse annealing at room temperature.

Acknowledgements: The research was supported and financed by European Union's Horizon 2020 Research and Innovation program under Grant Agreement no. 654168. The authors would like to thank Alexander Dierlamm for the proton irradiations at the Karlsruhe Institute of Technology (KIT), supported by the Initiative and Networking Fund of the Helmholtz Association, contract HA101(Physics at the Terascale) and the European Commission under the FP7 Research Infrastructures project AIDA, Grant agreement no.262025.

\section{REFERENCES}

1. G. Appolinari et al., High-Luminosity Large Hadron Collider (HL-LHC): Technical Design Report V. o.1, 
CERN Yellow Reports 226, CERN, Geneva, Switzerland, 2017.

DOI: 10.23731/CYRM-2017-004

2. Technical Design Report for the ATLAS Inner Tracker Strip Detector, Tech. Rep. CERN-LHCC-2017-005. ATLAS-TDR-025, CERN, Geneva, Switzerland, 2017. Retrieved from: https://cds.cern.ch/record/2257755/fil es/ATLAS-TDR-025.pdf;

Retrieved on: Aug. 10, 2018

3. G. Aad et al., "The ATLAS Experiment at the CERN Large Hadron Collider," J. Instrum., vol. 3, no. 8, So8003, Aug. 2008.

Retrieved from: http://iopscience.iop.org/article/10.108 8/1748-0221/3/08/So8003/pdf;

Retrieved on: Aug. 10, 2018

4. G. Lindstrom et al., "Radiation hard silicon detectors developments by the RD48 (ROSE) Collaboration," Nucl. Instr. Methods Phys. Res. A, vol. 466, no. 2, pp. $308-326$, Jul. 2011. DOI: 10.1016/So168-9002(01)00560-5

5. G. Casse, P. Allport, M. Hanlon, "Improving the radiation hardness properties of silicon detectors using oxygenated n-type and p-type silicon," IEEE Trans. Nucl. Sci., vol. 47, no. 3, pp. 527 - 532, Jun. 2000.

DOI: $10.1109 / 23.856475$

6. V. Cindro, G. Kramberger et al., "Radiation damage in p-type silicon irradiated with neutrons and protons," Nucl. Instr. Methods Phys. Res. A, vol. 599, no. 1, pp. 6o - 65, Feb. 2009.

DOI: 10.1016/j.nima.2008.11.007

7. G. Casse, P. P. Allport, A. Watson, "Effects of accelerated annealing on p-type silicon micro-strip detectors after very high doses of proton irradiation," Nucl. Instr. Methods Phys. Res. A, vol. 568, no. 1 , pp. $46-50$, Nov. 2006.

DOI: 10.1016/j.nima.2006.05.200

8. G. Kramberger et al., "Annealing studies of effective trapping times in silicon detectors," Nucl. Instr. Methods Phys. Res. A, vol. 571, no. 3, pp. 608 - 611, Feb. 2007.

DOI: 10.1016/j.nima.2006.10.399

9. M. Minano et al., "Annealing studies of silicon microstrip detectors irradiated at high neutron fluences," Nucl. Instr. Methods Phys. Res. A, vol. 591, no. 1, pp. $181-183$, Jun. 2008.

DOI: 10.1016/j.nima.2008.03.051

10. Y. Unno et al., "Development of $\mathrm{n}^{+-i n-p}$ large-area silicon microstrip sensors for very high radiation environments - ATLAS12 design and initial results,"
Nucl. Instr. Meth. Phys. Res. A, vol. 765, pp. $80-90$, Nov. 2014.

DOI: 10.1016/j.nima.2014.06.086

11. Hamamatsu photonics official web page, Hamamatsu photonics, Iwata City, Japan.

Retrieved from: https://www.hamamatsu.com/jp/en/in dex.html;

Retrieved on: Aug. 10, 2018

12. M. Mikestikova et al., "Study of surface properties of ATLAS12 strip sensors and their radiation resistance," in Proc. $10^{\text {th }}$ International "Hiroshima" Symposium on the Development and Application of Semiconductor Tracking Detectors (HSTD-1O), Xi'An, China, 2015 DOI: 10.1016/j.nima.2016.03.056

13. K. Hara et al., "Charge collection and field profile studies of heavily irradiated strip sensors for the ATLAS inner tracker upgrade," in Proc. 10 $0^{\text {th }}$ International "Hiroshima" Symposium on the Development and Application of Semiconductor Tracking Detectors (HSTD-1O), Xi'An, China, 2015. DOI: 10.1016/j.nima.2016.04.035

14. R. Mori et al., "Evaluation of the performance of irradiated silicon strip sensors for the forward detector of the ATLAS Inner Tracker Upgrade," in Proc. $10^{\text {th }}$ International "Hiroshima" Symposium on the Development and Application of Semiconductor Tracking Detectors (HSTD-1O), Xi'An, China, 2015. DOI: 10.1016/j.nima.2016.04.044

15. R. M. Hernandez, "A portable readout system for silicon microstrip sensors," Nucl. Instrum. Methods Phys. Res. A, vol. 623, no. 1, pp. 207 - 209, Nov. 2010. DOI: 10.1016/j.nima.2010.02.197

16. S. Löchner, M. Schmelling, The Beetle Reference Manual - Chip Version 1.3, 1.4 and 1.5, CERN, Geneva, Switzerland, 2006.

Retrieved from: http://inspirehep.net/record/928871/fi les/lhcb-2005-105.pdf?version $=1$; Retrieved on: May 22, 2018

17. M. Moll, "Radiation Damage in Silicon Particle Detectors," Ph.D. dissertation, University of Hamburg, Dept. of Physics, Hamburg, Germany, 1999. Retrieved from: https://mmoll.web.cern.ch/mmoll/thes is/pdf/moll-thesis.pdf; Retrieved on: Aug. 10, 2018

18. I. Mandic, V. Cindro, G. Kramberger, M. Mikuz "Annealing effects in $\mathrm{n}^{+-} \mathrm{p}$ strip detectors irradiated with high neutron fluences," Nucl. Instrum. Methods Phys. Res. A, vol. 629, no. 1, pp. 101 - 105, Feb. 2011. DOI: $10.1016 /$ j.nima.2010.11.057 